\title{
Hongos Filamentos de Relave Minero Contaminado con Plomo y Zinc
}

\author{
Filamentary Fungi of Mining Relay Contaminated with Lead and Zinc
}

Henry F. Llacza Ladera ${ }^{1}$, Pedro L. Castellanos Sánchez ${ }^{2}$

Recibido: Enero 2020 - Aprobado: Junio 2020 - Publicado: Junio 2020

\begin{abstract}
RESUMEN
El objetivo del trabajo fue identificar géneros de hongos filamentosos y determinar la concentración de plomo y zinc en las muestras de relave minero. Aislamos e identificamos hongos filamentosos mediante el método de diluciones seriadas y la técnica de micro cultivo respectivamente, también determinamos metales pesados, $\mathrm{pH}$ y el $\% \mathrm{H}$ de muestras de relave tomadas en tres áreas representativas. Identificamos nueve géneros fúngicos: Penicillium, Aspergillus, Paecylomices, Acremonium, Chrysosporium, Trichoderma, Alternaria, Helicosporium y Geoctrichum de un total de sesenta y dos cepas de hongos filamentosos presentes en el relave minero que presentaba las siguientes características físico químicas: Zinc (Zn) con 8810,4 ppm y plomo $(\mathrm{Pb}) 3844,4$ ppm, el pH tuvo una variación de 1,75-7,85 y \%H de 10,7 -2,6.
\end{abstract}

Palabras claves: Hongo; Relave; Género; Plomo; Zinc.

\begin{abstract}
The objective of the work was to identify genera of filamentous fungi and determine the concentration of lead and zinc in the mining tailings samples. We isolated and identified filamentous fungi using the serial dilution method and the micro culture technique respectively, we also determined heavy metals, $\mathrm{pH}$ and\% $\mathrm{H}$ of tailings samples taken in three representative areas. We identified nine fungal genera: Penicillium, Aspergillus, Paecylomices, Acremonium, Chrysosporium, Trichoderma, Alternaria, Helicosporium and Geoctrichum from a total of sixty-two strains of filamentous fungi present in the mining tailings that had the following physical-chemical characteristics: Zinc (Zn) with 8810.4 ppm and lead (Pb) $3844.4 \mathrm{ppm}$, the $\mathrm{pH}$ had a variation of $1.75-7.85$ and $\% \mathrm{H}$ of $10.7-2.6$.
\end{abstract}

Keywords: Fungi; Tailings; Genus; Lead; Zinc.

\footnotetext{
${ }_{1}$ Universidad Nacional Mayor de San Marcos, Laboratorio de Micología Aplicada, Facultad de Biología. Lima, Perú. E-mail: f6llacza@gmail.com

${ }^{2}$ Universidad Nacional Mayor de San Marcos, Laboratorio de Micología Aplicada, Facultad de Biología. Lima, Perú. E-mail: pcastellanoss@unmsm.edu.pe
} 


\section{INTRODUCCIÓN}

El relave minero es el residuo del proceso de concentración de minerales, usualmente son una mezcla de tierra, minerales, agua y roca que contienen altas concentraciones de químicos y metales ocasionando impactos negativos al ambiente durante y después de las actividades de extracción (Astete et al., s. f.) (Romero et al., 2008). Cuando se depositan los metales pesados en el suelo tienden a mantenerse en horizontes superficiales y si ocupan posiciones de intercambio catiónico son extraíbles por las plantas, constituyendo un grave problema al ecosistema (Cano, M., Moreno A., \& González, J., 1997).

El año 2005 el Banco Mundial emitió un informe sobre la problemática ambiental del sector minero en el Perú, realizando un ranking de las ciudades a nivel nacional con mayores pasivos ambientales mineros sin remediar, donde preocupantemente el distrito de Santa Bárbara de Carhuacayán, provincia de Yauli - Junin, presenta 20 pasivos ambientales que dañan la cuenca alta del río Mantaro (Diario Correo, 2012). Uno de estos pasivos ambientales es la desactivada unidad minera Carhuacayán, la cual detuvo sus actividades aproximadamente en el año 1980, esta depositó sus relaves mineros cerca al cauce del rio San Pedro, cuenca alta del rio Mantaro. Estas aguas son utilizadas en la zona para la ganadería.

En la naturaleza podemos encontrar microorganismos que toleran la exposición a metales pesados, dentro de estos podemos encontrar dos tipos de resistencia; los que poseen un sistema de detoxificación el cual es inducido por la presencia de metales pesados y los indiferentes a la presencia de metales, ambos son de interés debido a que presentan la característica de captar metales en ambientes contaminados. Se ha documentado dos formas de captar el metal, una es introduciendo el metal a nivel intracelular y la otra es adhiriendo el metal a la pared extracelular (Anahid et al., 2011).

Se ha documentado hongos filamentosos de los generos Trichosporon sp, Trichoderma sp, Penicillium sp, Aspergillus ps, Fusarium sp entre otros con la capacidad de tolerar y sorber metales pesados como plomo, zinc, cromo, cobre y tener la capacidad de captarlos hasta en un 64 \% (Muñoz et al., 2012), (Mazyar et al., 2010), (Bishnoi \& Nagpal, 2005). Pudiendo ser por quelación extracelular, intracelular o compartimentalización (Pócsi, 2011).

La efectividad en la sorción de metales pesados por hongos es debido a su disponibilidad, costo, regeneración y facilidad con la que se puede usar el biosorbente en varias configuraciones de reactores, factores importantes en la determinación del costo total de material biosorbente. Así, la biosorción ofrece una solución económica y opción técnicamente factible para el tratamiento de aguas residuales contaminadas con metales pesados (Kapoor \& Viraraghavan, 1995). Para la sorción de metales pesados empleando biomasa fúngica se emplea reactores de cama empacada, fluidizada, discos rotatorios y de lecho de lodos para esto los microorganismos tienen que formar biopeliculas en soportes preparados a partir de una variedad de materiales inertes, alguno de estos son: el agar, la celulosa, los alginatos, las poliacrilamidas, la sílica gel y el glutaraldehído (Gadd \& White, 1993). Algunas desventajas en el uso de biomasa fúngica, incluyen el tamaño de partícula, su baja resistencia mecánica y la dificultad para separar la biomasa del efluente.

En la actualidad existe muy poca información sobre el aislamiento e identificación de géneros fúngicos nativos de relave minero, por lo que el objetivo de nuestro estudio fue aislar e identificar géneros fúngicos $\mathrm{y}$ determinar la concentración de plomo, zinc y las condiciones fisicoquímicas del hábitat, relave minero.

\section{MATERIALES Y MÉTODO}

\subsection{Muestreo}

Desarrollamos el estudio en un relave que estuvo abandonado aproximadamente desde el año 1980 que perteneció a la unidad minera Carhuacayán, ubicada cerca al pueblo de Santa Bárbara de Carhuacayán, en el departamento de Junín, Perú. Empleamos la técnica de la cuadricula $(\mathrm{C})$ en tres zonas representativas del relave minero y se tomaron en total 15 muestras, cada zona con 5 muestras: C1:- CRH1, CRH2, CRH3, CRH4, CRH5; C2: CRH6, CRH7, CRH8, CRH9, CRH10; C3 - CRH11, CRH12, CRH13, CRH14, CRH15.

De cada cuadricula $(\mathrm{C})$ realizamos compósitos $(\mathrm{Co}): 1$, 2 y 3 y cada uno fue analizado por ICP-MS (Espectrometría de Masas con Plasma Acoplado Inductivamente) para determinar la concentración de metales pesados.

\subsection{Aislamiento e identificación de hongos filamentosos}

Empleamos el método de diluciones seriadas, método validado y útil para la caracterización inicial de la ecología de las comunidades fúngicas del suelo, disolvimos 10 gramos de muestra en $90 \mathrm{ml}$ de agua destilada estéril, agitamos durante $30 \mathrm{~min}$ a $160 \mathrm{rpm}$ en un agitador orbital marca GEMMY, diluimos de $10^{-1}$ hasta $10^{-4}$ e inoculamos en proporción 1:10 una cantidad de $50 \mathrm{ml}$ en cultivo líquido conteniendo glucosa, peptona y extracto de levadura (YPG), luego se colocó en un agitador marca GEMMY a 120 rpm durante 48 horas y se dejó en reposo por 6 días a temperatura de laboratorio. Con un asa de siembra los pelets formados por los hongos fueron sembrados en agar extracto de levadura, peptona, glucosa (Adaptado de Arias y Piñeros, 2008), luego se repicaron en agar papa dextrosa hasta obtener cultivos puros y conservados en viales con agar papa dextrosa (APD). Empleamos la técnica del micro cultivo y observaciones de las estructuras reproductivas para la identificación de géneros (Barnett y Hunter, s. f.).

\subsection{Determinación del pH y \% Humedad}

Empleamos un pH metro de mano marca ADWA para realizar mediciones de las muestras de $\mathrm{C} 1, \mathrm{C} 2$ y $\mathrm{C} 3$, mezclamos relave y agua destilada estéril en una proporción de $(1: 1)$, agitamos la mezcla y se deja reposar por dos horas para luego medir el valor de $\mathrm{pH}$.

Medimos el porcentaje de humedad mediante el método gravimétrico de las muestras $\mathrm{C} 1, \mathrm{C} 2$ y $\mathrm{C} 3$, para lo cual pesamos las muestras en una balanza analítica de 
precisión marca Shimadzu, secamos a $105^{\circ} \mathrm{C}$ en una estufa Nanbei, por 24 horas y finalmente se volvimos a pesar (peso seco) para luego calcular la humedad volumétrica mediante la siguiente fórmula:

H\% =Peso húmedo $(\mathrm{g})-$ Peso seco $(\mathrm{g}) /$ Peso seco $(\mathrm{g}) * 100$

\section{RESULTADOS}

\subsection{Géneros fúngicos identificados}

Identificamos 9 géneros fúngicos: Penicillium, Aspergillus, Paecylomices, Acremonium, Chrysosporium, Trichoderma, Alternaria, Helicosporium y Geoctrichum de 62 cepas fúngicas aisladas. Trabajamos las 15 muestras de relave en tres grupos: Cuadricula 1 (23 cepas), Cuadricula 2 (12 cepas) y Cuadricula 3 (27 cepas), Tablas 1 y 2.

Tabla 1. Géneros y cepas de hongos aislados en las cuadriculas (C) 1,2 y 3

\begin{tabular}{cccc}
\hline $\begin{array}{c}\text { Cuadricula } \\
\text { (C) }\end{array}$ & Genero & $\mathbf{N}^{\circ}$ de cepas & TOTAL \\
\hline \multirow{4}{*}{ C 1 } & Penicillium & 12 & \\
& Acremonium & 7 & 23 \\
& Alternaria & 3 & \\
& Aspergillus & 1 & \\
C 2 & Aspergillus & 5 & 12 \\
& Trichoderma & 4 & \\
& Penicillium & 3 & \\
C 3 & Penicillium & 10 & \\
& Paecylomices & 8 & \\
& Chrysosporium & 5 & \\
& Aspergillus & 2 & \\
& Helicosporium & 1 & \\
& Geoctrichum & 1 & \\
\hline
\end{tabular}

Tabla 2. Géneros fúngicos identificados de muestras de relave minero

\begin{tabular}{ccc}
\hline $\mathbf{N}^{\circ}$ & GENERO & $\mathbf{N}^{\circ}$ Cepas \\
\hline 1 & Penicillium & 25 \\
2 & Aspergillus & 8 \\
3 & Paecylomices & 8 \\
4 & Acremonium & 7 \\
5 & Chrysosporium & 5 \\
6 & Trichoderma & 4 \\
7 & Alternaria & 3 \\
8 & Helicosporium & 1 \\
9 & Geoctrichum & 1 \\
& TOTAL & 62 \\
\hline
\end{tabular}

3.2 Concentración de Zinc y Plomo en relave minero

Las concentraciones de Zinc y Plomo en ppm para el Compósito 1, Compósito 2 y Compósito 3 fueron de 9088,2; 3420,8 - 5375.8; 3789.3 y 11967.1; 4323.0 respectivamente, se observa una concentración mayor para el metal Zinc en comparación al metal Plomo (Tabla 3).

Tabla 3. Metales pesados Zinc y Plomo en Compósito 1, Compósito 2 y Compósito 3 analizados por ICP-MS

\begin{tabular}{ccccc}
\hline \multicolumn{5}{c}{ Unidad Minera Carhuacayan } \\
\hline $\begin{array}{c}\text { Metal } \\
\text { Pesado }\end{array}$ & $\begin{array}{c}\text { Compósito } \\
\text { 1 ppm }\end{array}$ & $\begin{array}{c}\text { Compósito } \\
\text { 2 ppm }\end{array}$ & $\begin{array}{c}\text { Compósito } \\
\text { 3 ppm }\end{array}$ & $\begin{array}{c}\text { Promedio } \\
\text { ppm }\end{array}$ \\
\hline Zinc (Zn) & 9088.2 & 5375.8 & 11967.1 & 8810.4 \\
Plomo (Pb) & 3420.8 & 3789.3 & 4323.0 & 3844.4 \\
\hline
\end{tabular}

$$
1 \mathbf{p p m}=1 \mathbf{m g} / \mathbf{k g} \text {. }
$$

\subsection{Determinación del pH y Humedad}

Los valores de $\mathrm{pH}$ para la cuadricula 1 , se encuentran entre 7,80 y 7,85 con un promedio de 7,80 la humedad presenta una variación que va entre 2,9 y 8,3 con un promedio de 4,5 de la misma forma se determinó los valores para la cuadricula 2 encontrando los valores de $\mathrm{pH}$ que varían entre 1.75 y 1.93 con promedio de 1.79 humedad entre 7,7 y 10,7 con promedio de 9,1 y la cuadricula 3 con $\mathrm{pH}$ entre 7.33 y 7.78 con un promedio de 7.60 y por último la Humedad entre 2,6 y 6,7 con promedio de 5,0. Se aprecia valores de $\mathrm{pH}$ neutros y ácidos, con bajo porcentaje de humedad (Tabla 4).

Tabla 4. Valores de porcentaje de humedad y pH en muestras de las cuadriculas (C) 1,2 y 3

\begin{tabular}{|c|c|c|c|c|c|}
\hline \multicolumn{6}{|c|}{ Unidad Minera Carhuacayan } \\
\hline $\begin{array}{l}\text { Cuadricula } \\
\text { (C) }\end{array}$ & MUESTRA & $\mathrm{pH}$ & $\begin{array}{c}\text { Promedio } \\
\text { pH }\end{array}$ & $\% \mathrm{H}$ & $\underset{\% \mathrm{H}}{\text { Promedio }}$ \\
\hline \multirow{5}{*}{ C 1} & CHR 1 & 7.80 & \multirow{5}{*}{7.80} & 2.9 & \multirow{5}{*}{4.5} \\
\hline & CHR 2 & 7.73 & & 3.5 & \\
\hline & $\mathrm{CHR} 3$ & 7.81 & & 4.6 & \\
\hline & $\mathrm{CHR} 4$ & 7.82 & & 8.3 & \\
\hline & CHR 5 & 7.85 & & 3.2 & \\
\hline \multirow{5}{*}{ C 2} & CHR 6 & 1.76 & & 8.9 & \multirow{5}{*}{9.1} \\
\hline & $\mathrm{CHR} 7$ & 1.75 & & 10.7 & \\
\hline & CHR 8 & 1.77 & 1.79 & 9.1 & \\
\hline & $\mathrm{CHR} 9$ & 1.75 & & 7.7 & \\
\hline & CHR 10 & 1.93 & & 9.0 & \\
\hline \multirow{5}{*}{ C 3} & CHR 11 & 7.33 & \multirow{5}{*}{7.60} & 6.7 & \multirow{5}{*}{5.0} \\
\hline & CHR 12 & 7.40 & & 6.6 & \\
\hline & CHR 13 & 7.78 & & 3.6 & \\
\hline & CHR 14 & 7.77 & & 5.7 & \\
\hline & CHR 15 & 7.74 & & 2.6 & \\
\hline
\end{tabular}

\section{DISCUSIÓN}

Identificamos nueve géneros fúngicos: Penicillium, Aspergillus, Paecylomices, Acremonium, Chrysosporium, Trichoderma, Alternaria, Helicosporium y Geoctrichum, de 62 cepas fúngicas aisladas obtenidas de relave minero, Tabla 2. Géneros como Aspergillus, Fusarium e Hypocrea también fueron aislados de relave de mina (Kumari et al., 2015). Podemos notar que el género Aspergillus es identificado también por otros autores lo que indica que este género tiene una buena capacidad de resistencia. Fomitopsis, Trichoderma, Rhizopus, Penicillium, 
Saccharomyces, Rhodosporidium y Aspergillus fueron identificados en suelo de mina (Oladipo et al., 2018); (Joo \& Hussein, 2012). Trichoderma y Aspergillus fueron encontrados en suelo industrial contaminado con Zinc (Mazyar et al., 2010); (Khamesy et al., 2016). Podemos notar que hay una diferencia entre la cantidad de géneros aislados en este trabajo en comparación a los que han sido revisados lo cual podría estar relacionado con el método de aislamiento o las condiciones ambientales de las muestras.

Nuestros resultados muestran concentraciones de zinc (8810.4 ppm) y plomo (3844.4 ppm) Tabla 3. Resultados semejantes encontraron (Joo \& Hussein, 2012) quienes analizaron muestras de suelo en área minera encontrando; Zinc (4383 ppm) y Plomo (3521 ppm). Los valores tienden a descender al analizar otro tipo de muestra como sedimento de rio contaminado con Zinc (219 ppm) (Mazyar et al., 2010), vertimientos de industrias como fertilizantes, energía eléctrica, mármol-granito y alimentos, plomo $(0.4528 ; 0.4224 ; 0.6652 ; 0.5390 \mathrm{ppm})$ y zinc $(0.223$; $0.225 ; 0.204 ; 0.306 \mathrm{ppm}$ ) respectivamente (Migahed et al., 2015). La influencia negativa de metales pesados sobre las poblaciones de microorganismos del suelo ha sido ampliamente descrita, así como la consecuente aparición de poblaciones adaptadas (Cervantes, 2006). Cepas fúngicas aisladas de sustratos con alta concentración de metales pesados exhiben características promisorias en recuperación de metales pesados (E. Jansen, M. Michels, M. van Til \& P. Doelman, s. f.).

El $\mathrm{pH}$ de los suelos alrededor de las minas tienden a variar entre 4.7 - 8.1 producto de sus actividades (Joo \& Hussein, 2012). La variación de $\mathrm{pH}$ encontrada para las muestras evaluadas fue de 1.75 a 7.80. Nuestros resultados presentan valores bajos de $\mathrm{pH}$ para las muestras del $\mathrm{C} 2$ llegando a valores de 1.75 Tabla 4 y valores neutros para $\mathrm{C} 1$ y $\mathrm{C} 3$. Los hongos presentes en $\mathrm{C} 2$ prometen futuros estudios debido a los valores bajos de $\mathrm{pH}$ el cual promueve la alta disponibilidad de los metales pesados constituyendo un ambiente estrés intenso para cualquier organismo. En comparación con otras actividades económicas como fundiciones industriales el $\mathrm{pH}$ oscila entre 6.9-9.24 (Qayyum et al., 2016).
Las condiciones de $\mathrm{pH}$ para el crecimiento de los hongos oscilan entre 4.5 y 8.0 es decir ligeramente ácidos, además tienden a acidificar el medio donde habitan para un mejor desempeño de su aparato enzimático llegando a $\mathrm{pH}$ menores a 2, por lo que pueden establecerse en ambientes muy ácidos (Mohammed \& Abdu, 2016) encontraron que la solubilidad del $\mathrm{Pb}, \mathrm{Cd}$ y $\mathrm{Zn}$ fueron más elevadas a $\mathrm{pH}$ entre 4,8 y 6,6 . El $\mathrm{pH}$ es uno de los principales factores que afecta inversamente proporcional la disponibilidad de la mayoría de los metales pesados, afectando la solubilidad, adsorción e intercambio de iones (Mc Bride et al., 1997), (Harter, 1983). Con excepción del Mo, Se y As, la biodisponibilidad de los metales pesados aumenta al mostrar valores bajos y disminuye con el aumento del pH del suelo (Basta \& Tabatabai, 1992), (Azevedo et al., 2003). La tolerancia de los hongos a $\mathrm{pH}$ bajo abren una perspectiva interesante en el área de la biosorción debido a que estas condiciones propician una mayor disponibilidad de metales pesados para ser recuperados.

El crecimiento de los microorganismos es influenciado por el porcentaje de la humedad relativa del sustrato en donde se encuentran (Ramos Vásquez \& Zúñiga Dávila, 2008). Para el desarrollo óptimo de los hongos es recomendable que la humedad relativa se encuentre a $70 \%$. En comparación a la humedad relativa de nuestras muestras oscilaron entre $2.6 \%$ y $10.7 \%$, Tabla 4 . La humedad encontrada es una condición que limita el crecimiento de las población fúngica establecida por ser ambientes secos para su desarrollo, los reportes de sustratos contaminados por metales pesados donde se hayan determinado la humedad relativa son escasos por lo este trabajo serviría de antecedente (Ver figura 1 y figura 2).

\section{CONCLUSION}

Identificamos nueve géneros fúngicos: Penicillium, Aspergillus, Paecylomices, Acremonium, Chrysosporium, Trichoderma, Alternaria, Helicosporium y Geoctrichum en 15 muestras de relave minero que presentaban valores elevados de plomo y zinc, en condiciones de $\mathrm{pH}$ ácido y humedad relativa baja. Estas características revelan

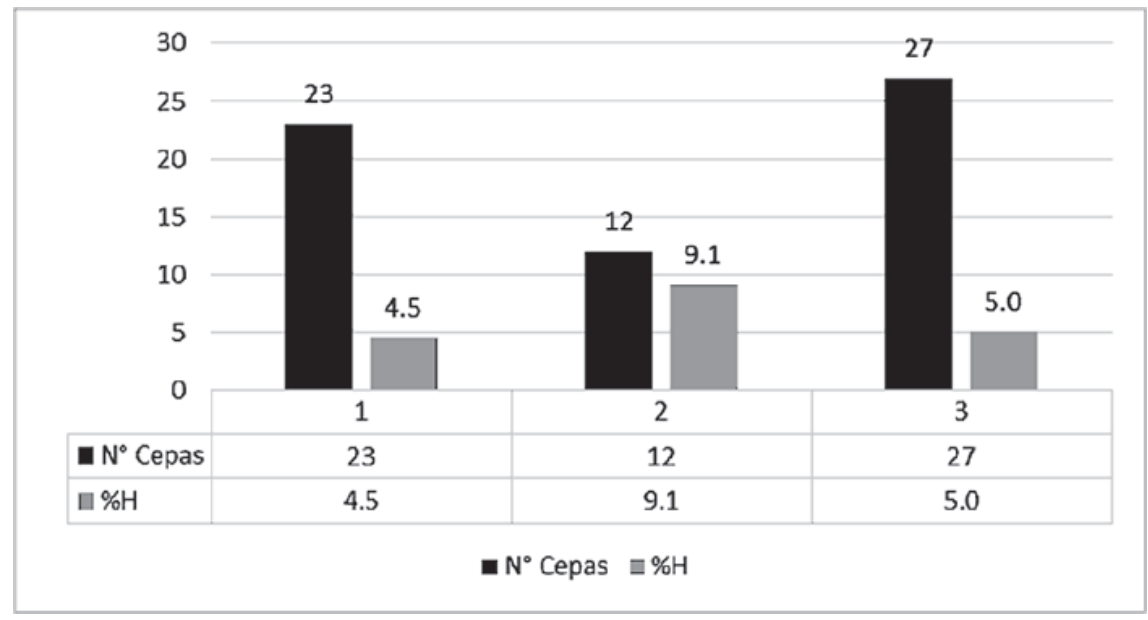

Figura 1. Relación entre humedad $(\% \mathrm{H})$ y cepas aisladas en las tres cuadrículas 1,2 y 3 . 


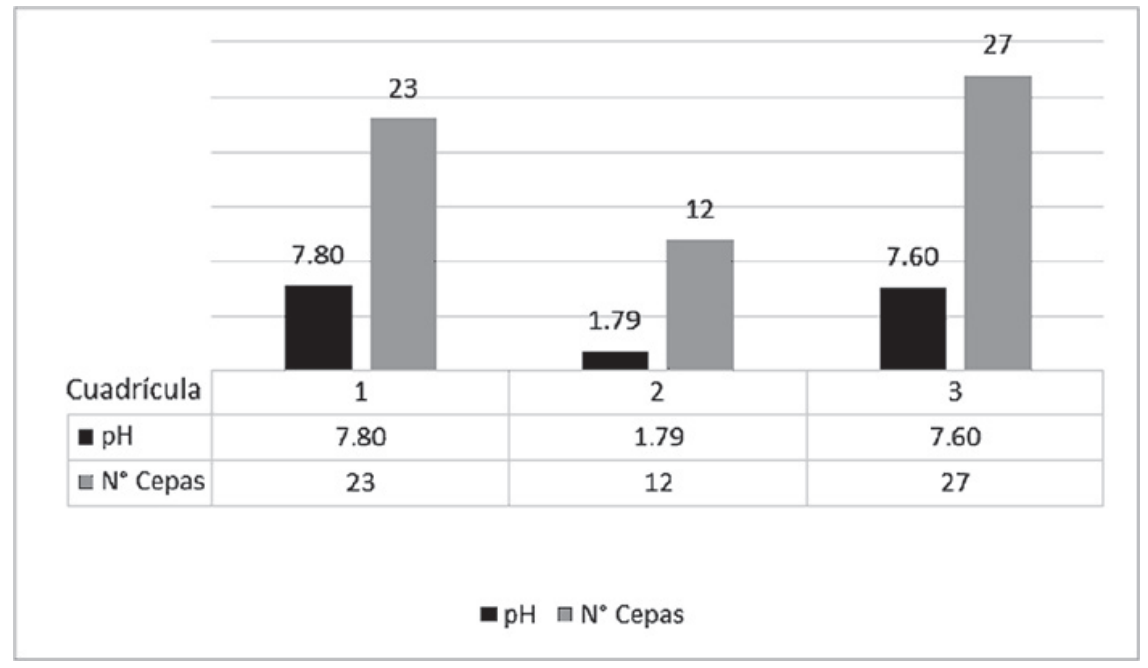

Figura 2. Relación entre pH y cepas aisladas en las cuadricula 1, 2 y 3

Tabla 5. Factores fisicoquímicos y cantidad de cepas aisladas

\begin{tabular}{cccccc}
\hline \multicolumn{5}{c}{ Unidad Minera Carhuacayan } \\
\hline $\begin{array}{c}\text { Cuadricula } \\
\text { (C) }\end{array}$ & Zinc (Zn)/ ppm & Plomo (Pb)/ ppm & pH & \% H & $\mathbf{N}^{\circ}$ Cepas \\
\hline C 1 & 9088.2 & 3420.8 & 7.80 & 4.5 & 23 \\
C 2 & 5375.8 & 3789.3 & 1.79 & 9.1 & 12 \\
C 3 & 11967.1 & 4323.0 & 7.60 & 5.0 & 27 \\
\hline
\end{tabular}

una capacidad de resistencia de los géneros nativos en nuestro país y amerita continuar los estudios para determinar su potencial en el campo de la biosorción de metales pesados.

\section{REFERENCIAS}

Anahid, S., Yaghmaei, S., \& Ghobadinejad, Z. (2011). Heavy metal tolerance of fungi. Scientia Iranica, 18(3), 502-508. https://doi.org/10.1016/j.scient.2011.05.015

Astete, J., Cáceres, W., Sabastizagal, I., Oblitas, T., Pari, J., \& Rodríguez, F. (s. f.). INTOXICACIÓN POR PLOMO Y OTROS PROBLEMAS DE SALUD EN NIÑOS DE POBLACIONES ALEDAÑAS A RELAVES MINEROS Rev Peru Med Exp Salud Publica., 5.

Azevedo, M. L. A., Alleoni, L. R. F., \& Guilherme, L. R. G. (2003). Biosolids and heavy metals in soils. Scientia Agricola, 60(4), 793-806. https://doi.org/10.1590/S010390162003000400029

Barnett y Hunter. (s. f.). Recuperado 7 de junio de 2020, de https://www.academia.edu/35499449/Illustrated genera of_imperfect_fungi-fourth_edition._Barnett_y_Hunter. pdf.pdf

Basta, N. T., \& Tabatabai, M. A. (1992). Effect of cropping systems on adsorption of metals by soils: II. Effect of pH. Soil Science; (United States), 153:3. https://doi org/10.1097/00010694-199203000-00004

Bishnoi, N., \& Nagpal, G. (2005). Bishnoi, N., \& Garima, N. Journal of Scientific and Industrial Research, 64. https:// www.researchgate.net/publication/267548113 Fungus An_alternative_for_bioremediation_of_heavy_metal containing_wastewater_A_review

Cano, M., Moreno A., \& González, J. (1997). Evaluación de la contaminación por metales pesados para suelos de cultivo. Ecología. 11: 83 - 89.

Cervantes, C. (2006). Interacciones microbianas con metales pesados. Rev Latinoam Microbiol, 8.

Diario Correo, R. (2012, enero 21). Carhuacayán en La Oroya en 4to. Lugar de pueblos contaminados. Correo. https:// diariocorreo.pe/peru/carhuacayan-en-la-oroya-en-4tolugar-de-pueblos-contaminados-502623/

E. Jansen, M. Michels, M. van Til \& P. Doelman. (s. f.). Recuperado 7 de junio de 2020, de https://link.springer.com/ article/10.1007/BF00336319

Gadd, G. M., \& White, C. (1993). Microbial treatment of metal pollution-A working biotechnology? Trends in Biotechnology, 11(8), 353-359. https://doi. org/10.1016/0167-7799(93)90158-6

Harter, R. D. (1983). Effect of Soil pH on Adsorption of Lead, Copper, Zinc, and Nickel. Soil Science Society of America Journal, 47(1), 47-51. https://doi.org/10.2136/ sssaj1983.03615995004700010009x

Joo, J.-H., \& Hussein, K. A. (2012). Heavy Metal Tolerance of Fungi Isolated from Contaminated Soil. Korean Journal of Soil Science and Fertilizer, 45(4), 565-571. https://doi. org/10.7745/KJSSF.2012.45.4.565 
Kapoor, A., \& Viraraghavan, T. (1995). Fungal biosorption an alternative treatment option for heavy metal bearing wastewaters: A review. Bioresource Technology, 53(3), 195206. https://doi.org/10.1016/0960-8524(95)00072-M

Khamesy, S. J., Hamidian, A. H., \& Atghia, O. (2016). Identification of the fungi absorbing heavy metals isolated from waste deposits of zinc factories. Mycologia Iranica, 3(1), 65-73. https://doi.org/10.22043/mi.2017.42384.1070

Kumari, D., Pan, X., Achal, V., Zhang, D., Al-Misned, F. A., \& Golam Mortuza, M. (2015). Multiple metal-resistant bacteria and fungi from acidic copper mine tailings of Xinjiang, China. Environmental Earth Sciences, 74(4), 3113-3121. https://doi.org/10.1007/s12665-015-4349-z

Mazyar, Y., Kong, Y., Abdullah, F., \& Tan, S. (2010). An in vitro Study on the Adsorption, Absorption and Uptake Capacity of $\mathrm{Zn}$ by the Bioremediator Trichoderma atroviride. EnvironmentAsia. https://doi.org/10.14456/ea.2010.8

Mc Bride, M., Sauve, S., \& Hendershot, W. (1997). Solubility control of $\mathrm{Cu}, \mathrm{Zn}, \mathrm{Cd}$ and $\mathrm{Pb}$ in contaminated soils. European Journal of Soil Science, 48(2), 337-346. https:// doi.org/10.1111/j.1365-2389.1997.tb00554.x

Migahed, F., Fawzy, G., \& Elrazak, A. A. (2015). Isolation of Heavy Metal Tolerant Fungi from Industrial Discharge. 19.

Mohammed, I., \& Abdu, N. (2016). Adsorption-Solubility Equilibiria and Speciation of $\mathrm{Pb}, \mathrm{Cd}$, and $\mathrm{Zn}$ in a Savanna Soil. https://www.researchgate.net/publication/310607276 Adsorption-solubility_equilibria_and_speciation_of_Pb_ $\mathrm{Cd}$ and $\mathrm{Zn}$ in a savanna soil
Muñoz, A., Ruiz, E., Abriouel, H., Gálvez, A., Ezzouhri, L., Lairini, K., \& Espínola, F. (2012). Heavy metal tolerance of microorganisms isolated from wastewaters: Identification and evaluation of its potential for biosorption. Chemical Engineering Journal, 210, 325-332. https://doi. org/10.1016/j.cej.2012.09.007

Oladipo, O. G., Awotoye, O. O., Olayinka, A., Bezuidenhout, C. C., \& Maboeta, M. S. (2018). Heavy metal tolerance traits of filamentous fungi isolated from gold and gemstone mining sites. Brazilian Journal of Microbiology, 49(1), 29-37. https://doi.org/10.1016/j.bjm.2017.06.003

Pócsi, I. (2011). Toxic Metal/Metalloid Tolerance in Fungi-A Biotechnology-Oriented Approach. En G. Banfalvi (Ed.), Cellular Effects of Heavy Metals (pp. 31-58). Springer Netherlands. https://doi.org/10.1007/978-94-007-0428-2 2

Qayyum, S., Khan, I., Maqbool, F., Zhao, Y., Gu, Q., \& Peng, C. (2016). Isolation and characterization of heavy metal resistant fungal isolates from Industrial soil, China. Pakistan journal of zoology, 48. https://www.researchgate.net/ publication/297732291 Isolation and characterization of_heavy_metal_resistant_fungal_isolates_from Industrial_soil_China

Ramos Vásquez, E., \& Zúñiga Dávila, D. (2008). Efecto de la humedad, temperatura y $\mathrm{pH}$ del suelo en la actividad microbiana a nivel de laboratorio. Ecología Aplicada, 7(12), 123-130.

Romero, A., Medina, R., \& Flores, S. (2008). Estudio de los metales pesados en el relave abandonado de Ticapampa. Revista del Instituto de Investigación de la Facultad de Ingeniería Geológica, Minera, Metalúrgica y Geográfica, 11(22), 13-16. 